\title{
Cabozantinib for progressive metastatic medullary thyroid cancer: a review
}

This article was published in the following Dove Press journal:

Therapeutics and Clinical Risk Management

28 May 2014

Number of times this article has been viewed

\author{
Joshua R Colombo \\ Richard O Wein \\ Department of Otolaryngology, Head \\ and Neck Surgery, Tufts Medical \\ Center, Boston, MA, USA
}

\begin{abstract}
Medullary thyroid cancer is uncommon and patients typically present with advanced disease. Treatment options for patients with progressive, metastatic medullary thyroid cancer had been limited until recently. Tyrosine kinase inhibitors have garnered increasing interest in this subset of patients. The US Food and Drug Administration recently approved cabozantinib, a tyrosine kinase inhibitor, after promising results were shown in a large Phase III clinical trial. This review summarizes the clinical pharmacology, clinical trials, and safety data for cabozantinib and concludes with a discussion of possible future directions for the treatment of medullary thyroid cancer.
\end{abstract}

Keywords: cabozantinib, tyrosine kinase inhibitor, medullary thyroid cancer

\section{Introduction}

Medullary thyroid carcinoma (MTC) is an uncommon neoplasm of the thyroid, representing approximately $5 \%$ of all thyroid malignancies, and has a prognosis unlike that of well differentiated thyroid carcinoma. MTC was first reported in the German literature in 1906 and described as a "malignant goiter with amyloid", reflecting the histopathologic appearance of the tumor. ${ }^{1}$

The tumor arises from the neuroendocrine parafollicular calcitonin-producing cells that are predominantly located at the junction of the upper and middle third of each thyroid lobe. Calcitonin secretion from the tumor may contribute to diarrhea at the time of initial evaluation; however the exact mechanism is not well known and other possible mediators include prostaglandins, calcitonin gene-related peptide, and serotonin, among others. Most MTC presentations (80\%) are associated with a spontaneous mutational event and typically present in the fifth or sixth decade of life. Patients with sporadic MTC characteristically present with upper pole thyroid nodules. ${ }^{2}$

Individuals with inherited tumor syndromes associated with MTC, which occur in one in 30,000 patients, and typically present at an earlier age, such as the third or fourth decade of life, without a specific gender predilection. ${ }^{1}$ The three inherited forms of MTC are familial MTC, multiple endocrine neoplasia type 2A (MEN2A), and multiple endocrine neoplasia type $2 \mathrm{~B}$ (MEN2B). These variants result secondary to germline mutations in the rearranged during transfection (RET) proto-oncogene.

Clinical presentation can vary as a result by the type of MTC pattern, ie, sporadic or inherited. All familial forms of MTC and MEN2 are inherited in an autosomal dominant fashion. MEN2A is characterized by hyperparathyroidism and pheochromocytoma, while MEN2B is associated with pheochromocytoma. The risk of hyperparathyroidism
Correspondence: Joshua R Colombo Department of Otolaryngology, Head and Neck Surgery, Tufts Medical Center, 800 Washington St \#850, Boston, MA 02III, USA

Tel $+|61763687| \mid$

Fax +16176361479

Email jcolombo@tuftsmedicalcenter.org 
and pheochromocytoma must be considered once a diagnosis of MTC is established. Symptoms that may be associated with pheochromocytoma include headache, palpitations, tremors, anxiety, and diaphoresis. A 24-hour urine can be obtained to assess for catecholamines, vanillylmandelic acid, and metanephrine levels. ${ }^{3}$

In MEN2A, the signs or symptoms of hyperparathyroidism or pheochromocytoma rarely present before the findings leading to the diagnosis of MTC. Symptoms of upper aerodigestive tract compression or invasion, such as dysphagia, odynophagia, and hoarseness, are reported in up to $15 \%$. Metastatic cervical adenopathy can be seen in $70 \%-80 \%$ of patients at initial presentation, while distant metastases (lung, bone) are present in 5\%-10\% of patients prior to diagnosis. ${ }^{4}$

MTC displays a slow growth pattern. However, metastasis to the liver and regional nodal basins plagues patients with hereditary disease, precluding them from curative resection and emphasizing the need for novel therapeutic strategies for metastatic MTC. ${ }^{5}$

\section{Molecular biology in MTC}

In both sporadic and inherited MTC, mutations in the RET proto-oncogene play a key role in pathogenesis. The $R E T$ proto-oncogene is mapped to chromosome 10q11.2 and encodes a $120 \mathrm{kDa}$ transmembrane receptor tyrosine kinase that functions as a target for the glial-derived neurotrophic factor family of growth factors. Mutations in RET are implicated in $95 \%$ of cases of hereditary MTC and greater than half of all sporadic presentations. Somatic RET mutations are associated with increased rates of advanced disease at diagnosis and a compromised prognosis. ${ }^{5-8}$

Moura et al analyzed RET mutations in 51 cases of sporadic MTC and identified somatic mutations in $64.7 \%$ of specimens. When compared overall, patients who were $R E T$-positive demonstrated no statistically significant difference in clinical or pathologic findings from individuals who were RET-negative. However, in subgroup analysis, sporadic MTC cases with exon 15 and 16 mutations were found to have a higher number of lymph node metastases, multifocal tumors, persistent disease, detectable calcitonin at last assessment, and more frequently had stage IV disease. Given these findings, the authors suggested that screening for somatic RET mutations, to potentially identify the more aggressive variants early in care, may help in the overall management of this population. ${ }^{9}$

In addition to mutations in RET, a number of other mutations have been associated with the development of MTC. Vascular endothelial growth factor (VEGF) and its receptor (VEGFR) are key signaling pathways in many cancers that influence angiogenesis. Increased expression of VEGF-A, VEGFR1, and VEGFR2 has been demonstrated in MTC $(50 \%$ of primary tumors, $75 \%$ of distant metastases). Mesenchymal-epithelial transition (MET) is a proto-oncogene that codes for the hepatocyte growth factor receptor, c-MET (a cell surface receptor), which is frequently mutated, overexpressed, or amplified in MTC. In normal tissue, the activated receptor stimulates a cascade that ultimately results in the promotion of cell division and motility, angiogenesis, and wound healing. An activated c-MET receptor promotes cell replication and reduces apoptosis (prolonging tumor cell survival and thus increasing malignant potential). ${ }^{6}$ The epidermal growth factor receptor is associated with regulation of cell growth, proliferation, and apoptosis. Epidermal growth factor receptor has been shown to be overexpressed in some cases of MTC. Tissue microassay studies found that only $20 \%$ of cases were strongly reactive for epidermal growth factor receptor and that tumors with the most aggressive RET mutations showed reduced epidermal growth factor receptor expression. ${ }^{10}$ Fibroblast growth factor receptor 4 has also been reported to be overexpressed in MTC. ${ }^{10}$

Examples of alternative genetic pathways important in the oncogenesis of MTC include the H-RAS mutations (in 56\% of RET-negative sporadic MTC) and activation of the mammalian target of rapamycin (mTOR) intracellular signaling pathway in hereditary MTC. ${ }^{11}$ The Ras-Raf-MEK-ERK pathway and its interaction with the phosphatidylinositol 3-kinase (PI3K)-AKT-mTOR pathway has also garnered significant research interest in MTC. Mutations in the Ras oncogene could play a role in the carcinogenesis of sporadic MTC. Ras operates in a complex signaling network with multiple activators and effectors, allowing them to regulate cell functions like proliferation, differentiation, apoptosis, and senescence. PI3K is one of the main effector pathways of Ras. Studies have reported a very high prevalence of Ras mutations in sporadic MTC, particularly RET-negative MTC. Sixty-eight percent of RET-negative MTCs had mutations of Ras compared with only $2.5 \%$ of RET-positive MTCs. ${ }^{10}$

\section{Diagnosis}

Patients with sporadic MTC typically present with a solitary thyroid nodule with or without palpable cervical lymphadenopathy. Diagnosis is made by fine needle aspiration, which demonstrates neuroendocrine cells and positive immunohistochemical staining for calcitonin, carcinoembryonic antigen (CEA), and chromogranin A. ${ }^{12}$ 
The initial evaluation for MTC per National Comprehensive Cancer Network (NCCN) recommendations includes a basal calcitonin level and CEA, pheochromocytoma screening, serum calcium, consideration for genetic counseling, screening for RET proto-oncogene mutation, neck ultrasound, and computed tomography (CT) with contrast of the chest. ${ }^{13}$

Basal calcitonin levels have been shown to correlate with tumor size and stage of disease. Calcitonin levels less than $100 \mathrm{pg} / \mathrm{mL}$ are associated with a median tumor size of $3 \mathrm{~mm}$ whereas levels greater than $2,000 \mathrm{pg} / \mathrm{mL}$ warrant additional imaging for metastatic disease. ${ }^{12} \mathrm{CEA}$ levels also provide information for risk stratification. Levels higher than $30 \mathrm{ng} / \mathrm{mL}$ were reported to be predictive of central and lateral cervical lymph node involvement in over $70 \%$ of patients. Levels greater than $100 \mathrm{ng} / \mathrm{mL}$ were associated with bilateral nodal disease and distant metastases. ${ }^{12}$ Loss of calcitonin expression and high CEA levels are markers of poorly differentiated and progressive disease. A favorable outcome and prognosis can be associated with an early age of diagnosis. ${ }^{11}$

\section{Surgical treatment}

Surgical management in MTC is dictated by the size and extent of disease. When the tumor is less than $1 \mathrm{~cm}$ in size, total thyroidectomy is recommended, with consideration for central neck dissection. When the tumor is greater than $1 \mathrm{~cm}$, total thyroidectomy with central neck dissection and consideration for prophylactic ipsilateral lateral neck dissection is advocated. Lateral neck dissection can be withheld for sporadic MTC when preoperative imaging fails to demonstrate regional lymphatic spread. There is only a $10 \%$ risk of lateral neck lymph node metastasis without the presence of central node metastases. However, the reported incidence of lateral neck spread increases to as high as $80 \%$ with involvement of one to three central nodes and is a near certainty with four or more positive central nodes. Despite this finding, controversy exists in the published literature concerning the performance of elective lateral neck dissection in patients with central neck lymph node metastasis. ${ }^{14}$

When incomplete resection of tumor occurs because of the extent of disease, adjuvant radiation therapy is advocated. In patients with distant metastatic disease, less aggressive surgical approaches appropriate to the specific presentation may be considered in an attempt to preserve function rather than empiric multilevel neck dissections. ${ }^{3}$

More than $50 \%$ of patients have a persistent elevation in calcitonin levels after their initial surgical management.
In selected cases, reoperative surgery for patients with persistently elevated calcitonin can result in biochemical normalization in approximately one quarter of patients and may achieve an improvement in overall survival. ${ }^{3}$

Patients with a family history of MTC should be considered for consultation for DNA testing to assess for RET mutation analysis. It is advocated that children with MEN2B and RET (codon $883,918,922$ ) mutations undergo total thyroidectomy in the first 6 months of life with a central compartment nodal dissection. Children with RET codon 611, 618, 620, 634 mutations are recommended to undergo total thyroidectomy before 5 years of age. Children with RET codon 609, 768, $790,791,804,891$ mutations are recommended to have total thyroidectomy with no specific age consensus stated. ${ }^{3}$

The 2009 American Thyroid Association management guidelines for MTC were published prior to the recent clinical trials of targeted therapy for progressive MTC. Recommended postoperative assessment of patients included initial basal calcitonin and CEA. With elevated calcitonin levels ( $>150 \mathrm{pg} / \mathrm{mL})$, systemic assessment may include neck ultrasound, neck and chest $\mathrm{CT}$, three-phase contrast-enhanced liver $\mathrm{CT}$ or magnetic resonance imaging, magnetic resonance imaging of the spine/pelvis, and bone scan. ${ }^{1}$

Five-year survival depends on stage. Stage I-II has a 5-year survival of $98 \%-100 \%$, while stage III and IV disease ranges from $81 \%$ to $23 \%$, respectively. MTC confined to the thyroid has a reported 10 -year survival rate of $95.6 \%$. This rate decreases to $75.5 \%$ in patients with regional spread of disease and to $40 \%$ in those with metastatic disease. ${ }^{15}$

\section{Chemotherapy for MTC}

Cytotoxic chemotherapy in MTC has poor response rates and a short duration of effect. There is no well accepted standard regimen with significant benefit in this setting. ${ }^{6}$ Doxorubicin has traditionally been the treatment of choice, but response is usually poor and the risk of toxicity is significant. ${ }^{7}$

\section{Radiation for MTC}

Since MTC arises from C cells, which do not concentrate iodine, radioactive iodine is ineffective as a treatment. External beam radiation therapy (EBRT) for MTC has been described, with indications including postoperative radiation in patients without distant metastatic spread with residual disease (gross or microscopic), extranodal disease with soft tissue extension, mediastinal spread, and persistent detectable postoperative calcitonin levels. ${ }^{16}$

EBRT has been described in the treatment of recurrent or metastatic MTC, but its use is uncommon. Martinez et al used 
the Surveillance, Epidemiology, and End Results database to identify a population with primary, histologically confirmed MTC treated with total thyroidectomy and one or more lymph nodes removed between 1988 and 2004. The final sample size included 534 patients, of which 66 received EBRT. In univariate analysis, EBRT was not associated with significant improvement in overall survival $(P<0.14)$. Multivariate analysis restricted to node-positive patients did not show improved overall survival with EBRT. ${ }^{17}$

The goal of adjuvant radiation is to improve locoregional control rates. Anatomic sites at unique risk for lifethreatening complications with local recurrence include the tracheoesophageal groove, carotid sheath, and retropharynx, and warrant strong consideration for adjuvant radiation when a risk of microscopic residual disease exists. In the setting of distant metastases, radiation to the neck could be considered if the palliative intent of stabilizing uncontrolled high-risk disease could prolong survival. ${ }^{13}$ However, conventional radiotherapy to this area can be associated with significant local toxicity.

\section{Recurrence}

When investigating possible recurrent or metastatic MTC, there is no single sensitive diagnostic imaging method. The conventional imaging methods (ultrasound, magnetic resonance imaging, CT) frequently fail to reveal recurrent lesions, and the sensitivity of ${ }^{131} \mathrm{I}-\mathrm{MIBG}$ (metaiodobenzylguanidine) or ${ }^{123} \mathrm{I}-\mathrm{MIBG}$ and Octreoscan ${ }^{\circledR}$ (the most used radiopharmaceuticals in neuroendocrine tumors) is low. Positron emission tomography CT using ${ }^{18} \mathrm{~F}-\mathrm{FDG}$ (fluorodeoxyglucose) is currently the best option for evaluating patients with persistent calcitonin elevation, as the sensitivity for recurrent or residual disease detection is reported to be $44.1 \%-85 \%{ }^{18}$ However, some studies have suggested that ${ }^{18} \mathrm{~F}$-DOPA (fluorine-18-L-dihydroxyphenylalanine) has better sensitivity then ${ }^{18}$ F-FDG. ${ }^{19,20}$

For patients with recurrent or persistent disease, locoregional or distant metastases, vandetanib and cabozantinib are category 1 NCCN recommendations. Category 1 recommendations are based on high-level evidence and there is uniform NCCN panel consensus that the intervention is appropriate. $^{13}$

\section{Cabozantinib: mechanism of action/initial reports}

Cabozantinib (XL184) is an oral tyrosine kinase inhibitor (TKI), also known as a multikinase inhibitor, with activity against $M E T$, VEGFR2, and RET. Activation of these recep- tors has been implicated in both development and progression of MTC. ${ }^{6,7}$

The US Food and Drug Administration approved cabozantinib (Cometriq ${ }^{\circledR}$, Exelixis, Inc., San Francisco, CA, USA) in November 2012 for metastatic MTC. It is orally bioavailable and patients are instructed to not eat for 2 hours prior to taking the drug and not to eat for one hour after. The recommended dose is $140 \mathrm{mg}$ daily (one $80 \mathrm{mg}$ capsule and three $20 \mathrm{mg}$ capsules). Metabolism occurs in the liver via cytochrome P450 (CYP)2C8. ${ }^{21}$ Cabozantinib has an acceptable safety profile, its half-life (91.3 \pm 33.3 hours) supports once-daily dosing, and it is active in patients who have progressed on other therapies, including other TKIs (such as vandetanib). ${ }^{21,22}$

Sennino et al compared the effects of cabozantinib, which blocks VEGFR and c-MET, with inhibition of VEGFR using a selective antibody in pancreatic neuroendocrine tumors. After treatment with anti-mouse $V E G F$ antibody for 3 weeks, tumors were smaller than vehicle-treated tumors but had greater expression of c-MET and more irregular borders. Irregularity indicative of invasion of the surrounding acinar pancreas was more numerous. Tumors treated with cabozantinib were not only smaller, but were less invasive and had no liver metastasis. All mice treated with cabozantinib survived until 20 weeks (primary end point) but no mice from either other group reached 20 weeks. This study confirmed that selective inactivation of $V E G F$ reduces tumor growth but leads to greater invasiveness and metastases. Inhibition of VEGFR and c-MET together slows tumor growth, decreases invasiveness, metastasis, and prolongs host survival. ${ }^{23}$

Yakes et al tested the selectivity of cabozantinib against a protein kinase panel of approximately 270 human kinases. This revealed potent inhibition of MET and VEGFR2, and of $K I T, R E T, A X L, T I E 2$, and Fms-like tyrosine kinase 3. A single $100 \mathrm{mg} / \mathrm{kg}$ dose of cabozantinib resulted in inhibition of MET and VEGFR2 phosphorylation 2-8 hours post dose. Yakes et al were able to show that cabozantinib had an antiangiogenic effect rather than a cytotoxic effect by showing that it inhibited endothelial cell tubule formation in vitro. Cabozantinib significantly increased tumor hypoxia and apoptosis at 8 and 4 hours after the first and second doses by disrupting tumor vasculature and promoting tumor and endothelial cell death. The impact of cabozantinib on metastasis compared with that of other VEGFR2-targeting therapies known to promote metastasis was also investigated in preclinical models, and evidence of inhibition of metastasis was seen. ${ }^{24}$

Bentzien et al performed in vitro biochemical and cellbased assays with cabozantinib and in vivo xenograft tumor 
studies in nude mice. Inhibition of oncogenic RET kinase activity and dose-dependent tumor growth inhibition was noted as a result of treatment with cabozantinib. In immunohistochemical analysis of tumor specimens, cabozantinib decreased phosphorylated $M E T$ and RET, tumor vascularization, and tumor cellularity. In vivo studies also demonstrated reduced secretion of calcitonin in MTC cells treated with cabozantinib. ${ }^{25}$

Many of the initial clinical reports on the utility of cabozantinib were focused on pathologies other than MTC. In a Phase II study, Smith et al reported on the use of cabozantinib (100 mg daily oral dosing) in castration-resistant prostate cancer and demonstrated improvement in progression-free survival and reduction of soft tissue tumors and bone turnover markers. Dramatic results seen on post-treatment bone scans suggested a direct effect of cabozantinib on tumor cells and the bone microenvironment not seen previously with $V E G F$ targeted therapies. ${ }^{26}$

RET fusions are present in 1\%-2\% of non-small-cell lung carcinoma cases, with an increasing incidence seen in neversmokers with lung adenocarcinoma. Drilon et al reported on preliminary data in three patients treated with cabozantinib in a prospective Phase II trial for patients with RET fusionpositive non-small-cell lung carcinoma (NCT01639508). ${ }^{27}$ Partial responses were observed in two patients, and all three patients remain progression-free on treatment. ${ }^{28}$

MTC can develop resistance to cytotoxic drugs due to the expression of the multidrug resistance (MDR)1 gene. The most promising results in MTC unresponsive to chemotherapy and radiation therapy have been seen with the TKIs. Inhibition of a single tyrosine kinase receptor can result in compensatory signaling that maintains cell growth. Multitargeted TKIs, such as cabozantinib, have been developed to bypass this potential resistance. Some patients cannot take advantage of these therapies due to specific RET mutations that confer resistance (RETV804 confers resistance to vandetanib). This type of resistance has not yet been reported with cabozantinib. ${ }^{10}$

\section{Clinical trials with cabozantinib and MTC}

In a Phase I trial of 25 patients with advanced stage tumors, Salgia et al showed that cabozantinib resulted in a substantial reduction in plasma calcitonin with associated stable disease for over 6 months in three patients with MTC. These researchers concluded that cabozantinib was well tolerated, with a long terminal half-life and antitumor activity observed at dosages not associated with toxicity. ${ }^{29}$
Kurzrock et al performed a Phase I open-label dose-escalation study of cabozantinib in patients with a wide range of advanced malignancies (including an expanded cohort with MTC). The study enrolled adult patients with confirmed solid tumors or lymphomas that were metastatic or unresectable and no longer responding to conventional therapies or who had disease for which no standard therapy existed $(n=85)$. Using a three-plus-three study design, patients were assigned to 13 dose levels exploring two different schedules of administration and formulations. The MTC cohort received $175 \mathrm{mg}$ using fixed daily dosing with capsules. Tumor response was assessed using Response Evaluation Criteria for Solid Tumors (RECIST) at baseline, 28 days after the first dose, and every 8 weeks thereafter. Response was confirmed by repeat imaging and pharmacokinetic, calcitonin, and CEA analyses. In this study, 77 patients with measurable disease were evaluable for response, and included 35 MTC patients. An objective response was achieved in ten of these 35 patients (with measurable disease), with 50\% showing a partial response at the first radiologic assessment. Overall, 17 patients experienced a 30\% or greater decrease in tumor measurements compared with baseline measurements. Stable disease (of at least 6 months' duration) was observed in 15 patients. Onset of response in the MTC group was as early as 21 days and as late as 365 days. Median time to response was 49.5 days. The median duration of response had not been reached at the time of reporting. Three of ten responses seen in the MTC group were in patients who had failed prior TKIs known to inhibit RET (vandetanib and sorafenib). Analysis of biomarkers showed reductions in serum calcitonin of $3 \%$ to $99 \%$ from baseline in 28 of 30 patients. A CEA reduction of $13 \%-94 \%$ was seen in 24 patients. $^{22}$

Of note, $90 \%$ of patients reported at least one treatment-related adverse event (AE), with $43 \%$ of AEs assessed as grade 1 or 2. The most frequent AEs were diarrhea, fatigue, decreased appetite, nausea, palmar-plantar erythrodysesthesia, rash, increased aspartate transaminase, vomiting, and mucositis. One grade 4 AE (pulmonary embolism) was reported and assessed to be related to cabozantinib. No grade 5 events or variation of AEs between tumor types were noted. ${ }^{22}$

This Phase I study demonstrated that cabozantinib was active in MTC, with an acceptable spectrum of toxicity. AEs were largely the same as those seen with other receptor tyrosine kinase inhibitors. The incidence of hypertension (16\% all grades, including two grade $3 \mathrm{AEs}$ ) was lower than with other TKIs (ie, motesanib and axitinib). The confirmed partial response rate of $29 \%$, rapidity of response, and prolonged duration of response observed in a largely pretreated MTC population compared favorably with 
efficacy reported in other trials of TKIs in MTC. Evidence of tumor regression was observed in patients with and without identified RET mutations. These results suggest that the anticancer effect was due to inhibition of targets other than RET (VEGFR2 and MET). Of note, 12 of 15 patients with somatic M918T mutation in RET had evidence of durable tumor shrinkage and stable disease (M918T is a known strong negative prognostic indicator). ${ }^{22}$ Responses were seen in patients regardless of the RET mutation status of their tumors, suggesting that the drug is active in patients without RET-activating mutations. ${ }^{29}$

The largest study of cabozantinib for MTC was a randomized Phase III study performed by Elisei et al. ${ }^{30}$ They conducted a double-blind trial in 330 patients with metastatic MTC comparing cabozantinib $140 \mathrm{mg}$ /day with placebo. Patients needed to have documented radiographic evidence of disease progression, no prior systemic cancer therapy 4 weeks prior to enrollment, but could have had prior TKIs in their care history. Randomization was 2:1 (cabozantinib to placebo) and patients were stratified by age and prior TKI treatment. The primary end point of the study was progression-free survival. ${ }^{30}$

The median age of the patients was 55 years. The main sites of metastatic disease were lymph nodes (79.9\%), liver $(69.4 \%)$, lung $(53 \%)$, and bone $(51.1 \%)$, with more than $85 \%$ of patients having involvement of two or more sites. Approximately $50 \%$ of patients were found to be $R E T$ mutation-positive, with M918T being the predominant $R E T$ mutation isolated in the group, while RET mutation status was unknown in $38.7 \%$ of the study patients. ${ }^{30}$

A statistically significant improvement in estimated median progression-free survival was seen in the cabozantinib group (11.2 months) versus placebo (4.0 months), with a stratified hazards ratio of 0.28 . Improvement in progression-free survival was seen across all group stratifications (age, prior treatment, and RET mutation status). Kaplan-Meier estimates of the percentage of patients progression-free and alive at one year were $47.3 \%$ for the cabozantinib group and $7.2 \%$ for the patients in the placebo arm. The response rate for cabozantinib was $28 \%(0 \%$ for placebo $){ }^{24}$

Side effects were substantial, requiring dose reductions in $79 \%$ of patients and discontinuation of therapy in $16 \%$. Adverse events reported included nausea, diarrhea, hypocalcemia, palmar-plantar erythrodysesthesia, hypertension, pulmonary embolism, weight loss, loss of appetite, and fatigue. Grade 3 or 4 adverse events were reported in $69 \%$ and $33 \%$ of patients respectively, with the most common being diarrhea $(15.9 \%)$ and palmar-plantar erythrodysesthesia
(12.6\%). Clinically relevant QT prolongation was not encountered in the trial. ${ }^{30}$

\section{Side effects of cabozantinib}

Beyond the AEs mentioned above, boxed warnings regarding perforation/fistula and hemorrhage are listed for this drug. Gastrointestinal fistulas are reported in 3\% of patients. Hemorrhage, with hemoptysis and/or gastrointestinal bleeding, was reported in 3\% under treatment and patients should be monitored for signs of bleeding while on therapy. For patients undergoing surgery, cabozantinib should be stopped at least 28 days prior because of the potential for wound healing complications.

Additional AEs reported in patients undergoing treatment with cabozantinib include the potential for thrombotic events (venous thromboembolism 6\%, arterial thromboembolism $2 \%$ ), treatment-emergent hypertension (61\%), osteonecrosis of the jaw ( $1 \%)$, proteinuria $(2 \%)$, and reversible posterior leukoencephalopathy syndrome. In one trial, palmar-plantar erythrodysesthesia syndrome was reported in 50\% of patients, with a grade 3 reaction noted in $13 \%$.

Cabozantinib should not be considered in patients with a history of hepatic impairment. Drug interactions have been reported with CYP3A4 inhibitors (such as ketoconazole, clarithromycin, and ritonavir) and CYP3A4 inducers (such as phenytoin, rifampin, and phenobarbital). ${ }^{31}$

\section{Other TKIs tested in MTC}

Vandetanib is a low molecular weight TKI that has demonstrated effective inhibition of RET in vitro. It blocks in vivo phosphorylation and signaling of the RET/MEN2B oncoprotein and prevented growth of two human cancer cell lines that carry spontaneous RET rearrangements. ${ }^{5}$ Vandetanib has activity against epidermal growth factor receptor, which cabozantinib does not.

In a Phase II open-label, single-arm study performed by Robinson et al, patients received vandetanib $100 \mathrm{mg}$ until disease progression, unacceptable toxicity, or withdrawal of consent. The primary aim of the study was to assess the objective response rate with vandetanib $100 \mathrm{mg} /$ day monotherapy according to RECIST. Additional assessments included progression-free survival, disease control rate, safety/tolerability, and biochemical response (serum levels of calcitonin and CEA). Nineteen patients with MTC who had undergone prior thyroidectomy were enrolled. Eighteen had metastatic disease and one had locally advanced disease with lymph node involvement. At data cutoff, eleven patients were still on vandetanib $100 \mathrm{mg} /$ day. Three patients had a partial 
response $(30 \%$ or greater decrease in sum of longest diameter measurements). Stable disease of 24 weeks or longer was seen in ten patients. Only two patients experienced adverse events causing interruption of treatment, one of whom

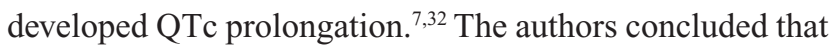
vandetanib $100 \mathrm{mg} /$ day demonstrated antitumor efficacy in patients with advanced or metastatic MTC. Therefore it may allow for dose decreases in patients who experience significant toxicity with $300 \mathrm{mg} /$ day. ${ }^{32}$

Wells et al conducted a randomized, double-blind Phase III trial of vandetanib in MTC. A total of 331 patients with MTC were enrolled (231 in the vandetanib arm, 100 in the placebo arm). At the time of analysis, 124 patients (37\%) had progressed and 48 (15\%) had died. Significant prolongation of progression-free survival was observed for patients on vandetanib compared with those on placebo. Median progression-free survival for the placebo group was 19.3 months. Median progression-free survival had not been reached for the vandetanib group, but using a Weibull model was predicted to be 30.5 months. Progression-free survival at 6 months was $83 \%$ (vandetanib) and 63\% (placebo). Thirtyone patients discontinued treatment during the randomized phase due to adverse reactions, including $28(12 \%)$ on vandetanib and three $(3 \%)$ on placebo. Nineteen $(8 \%)$ patients developed protocol-defined QTc prolongation. Five patients receiving vandetanib experienced adverse events leading to death. The results of this study showed that vandetanib has good efficacy in patients with MTC, with a largely manageable side effect profile. ${ }^{33}$

Vandetanib (Caprelsa ${ }^{\circledR}$, AstraZeneca, Wilmington, DE, USA) was approved in April 2011, but has significant toxicity. QT prolongation with torsades de pointes and sudden cardiac death have been reported in patients taking vandetanib. ${ }^{7}$ Vandetanib needs to be avoided in patients with cardiac disease, but this only accounts for a small subset of patients. ${ }^{7}$

Sorafenib is an oral small-molecule TKI that targets VEGFR2, VEGFR3, RET, and BRAF. In a study of 16 patients with advanced MTC (stratified into hereditary or sporadic) treated with sorafenib $400 \mathrm{mg}$ twice daily, one patient achieved a partial response and 14 attained stable disease, with four having stable disease lasting longer than 15 months. The median progression-free survival was 17.9 months. ${ }^{11,34,35}$

Motesanib is a TKI that targets VEGFR1, VEGFR2, and VEGFR3. In a study by Schlumberger et al, 91 patients with MTC were treated with $125 \mathrm{mg}$ of motesanib daily. Partial remission was seen in two patients, stable disease in $81 \%$, and median progression-free survival was 48 weeks. ${ }^{11,34,36}$
Sunitinib is an inhibitor of VEGFR (1, 2, and 3), RET, and RET/PTC subtypes 1 and 3. Goulart et al gave sunitinib $37.5 \mathrm{mg}$ daily to patients with FDG-avid, advanced thyroid cancers (seven MTC, 28 differentiated thyroid cancer), and three of six patients with MTC achieved a RECIST response. ${ }^{11,34,37}$

Axitinib is another inhibitor of the VEGF receptor. In a Phase II trial of axitinib for advanced thyroid carcinoma that included eleven MTC patients (18\% of the study population), Cohen et al reported a partial response rate of $18 \%$ and a stable disease rate ( $>24$ weeks) of $27 \%$. $^{11,34,38}$

In a study by de Groot et al, ${ }^{39} 15$ patients with confirmed MTC were treated using $600 \mathrm{mg}$ of imatinib daily. There were no objective responses and median duration of treatment was 4 months. Frank-Raue et $\mathrm{al}^{40}$ treated nine patients with MTC using $600 \mathrm{mg}$ of imatinib daily for a median duration of 13 months. Seven patients achieved stable disease at 3 months, but only one remained stable at 12 months. There were no clinical responses and median progression-free survival was 6 months. ${ }^{11,34,39,40}$

An in vitro study comparing four TKIs found cabozantinib to be the most potent inhibitor in MEN2A MTC and vandetanib most potent in MEN2B MTC. ${ }^{41}$ Patients who have failed vandetanib have shown a response to cabozantinib, but the opposite sequence has not been studied. In conclusion, cabozantinib is the first treatment shown to improve progressionfree survival in progressive MTC and offers clinicians a novel and effective tool to help patients with this disease. ${ }^{7}$

\section{Future uses and indications}

For advanced stage or recurrent MTC not amenable to curative surgical management, cabozantinib and vandetanib are treatment options with US Food and Drug Administration approval. The two Phase III trials investigating their use in this setting differed significantly and are not easily compared. Median progression-free survival was longer in the vandetanib arm of the ZETA trial ${ }^{42}$ (30.5 months, 11.2 months longer than placebo) than in the cabozantinib arm of the EXAM trial ${ }^{43}$ (11.2 months, 7.2 months longer than placebo), although the placebo arms differed markedly from each other. However, the ratio of median progression-free survival with cabozantinib's placebo arm was 2.80 , but was 1.58 with vandetanib, suggesting greater benefit with cabozantinib. Of note, one significant difference between the two drugs is the absence of significant QTc interval prolongation in patients treated with cabozantinib, so cabozantinib should be the treatment of choice in patients with a history of heart disease, arrhythmia, or QT prolongation. ${ }^{29}$ 
Verbeek et al compared the efficacy of cabozantinib, vandetanib, sunitinib, and axitinib using three different cell lines, ie, MTC-TT derived from a sporadic MTC expressing a C634W RET mutation (MEN2A mutation), MZ-CRC-1 derived from a metastatic sporadic MTC expressing a M918T RET mutation (MEN2B mutation), and TPC-1 derived from papillary thyroid cancer expressing a RET/ PTC-1 rearrangement. A dose-dependent decrease in cell proliferation was seen with all four TKIs. Cabozantinib was the most effective inhibitor of MTC-TT and TPC-1, while vandetanib was the most effective inhibitor of MZ-CRC-1. This in vitro study suggests that mutation-specific therapy could be beneficial for the treatment of MTC. ${ }^{41}$

In addition, as new pathways involved in the pathogenesis of MTC are elucidated and drugs to target these are developed, combination therapy may ultimately offer patients a survival benefit. As mentioned before, the Ras-Raf-MEKERK pathway and its interaction with the PI3K-AKT-mTOR pathway is associated with sporadic MTC. Everolimus has activity against the PI3K/AKT/mTOR pathway and has demonstrated antitumor efficacy in MTC. ${ }^{10}$ One could start to extrapolate that combination therapy with everolimus and cabozantinib might offer the potential for a survival benefit in MTC. Cabozantinib through apoptosis and everolimus through cell cycle arrest and senescence. ${ }^{24,44}$ However, further investigation is needed.

Starenki et al reported on the inhibition of the leukemia inhibitory factor-mediated autocrine/paracrine loop in human MTC xenografts in mice. To accomplish this, they used bacterially produced recombinant leukemia inhibitory factor and demonstrated growth suppression, activation of the JAK/STAT pathway, and downregulation of RET and E2F1 expression in tumors. This cytostatic form of oncogenic RET inhibition is cytokine-mediated and potentially complementary to existing approved therapies. Leukemia inhibitory factor has been used previously in the treatment of selected neurologic conditions (eg, amyotrophic lateral sclerosis) and has been shown to be relatively nontoxic in this clinical setting. ${ }^{45}$

Early preclinical studies of a new family of medications known as withanolides have also shown promise in MTC. The most common withanolide is withaferin A, which has been shown to decrease MTC tumor mass in vivo in a metastatic mouse model.

In a study by Samadi et al, withanolides impaired MTC cell growth through induction of apoptosis, promoted cell cycle arrest in MTC cells, promoted inhibition of clonogenic growth, and suppressed activation of RET and the AKT/mTOR pathway. These compounds may have a role in the future treatment of MTC but additional investigation is required. ${ }^{46}$

Our understanding of the molecular biology of MTC has advanced considerably, although surgery remains the most effective treatment modality. TKIs effecting RET are promising, but the absence of complete responses in monotherapy emphasizes the need for more active single agents or discovery of effective therapeutic combinations. Inhibition of RET may render these tumors more sensitive to chemotherapy, but to date no major combination trials have been published. ${ }^{47}$

\section{Summary}

New approaches to the preclinical development of targeted agents are necessary for patients with unresectable and recurrent MTC. The lack of curative chemotherapeutic interventions and the challenging side effect profiles of current treatment options mandate continued investigation. Although multikinase inhibitors have been reported to be effective in approximately $50 \%$ of MTC cases, their side effect profile frequently requires dose reduction and/or drug discontinuation in one quarter of patients treated. For patients with recurrent or persistent disease, or locoregional or distant metastases, vandetanib and cabozantinib are US Food and Drug Administration-approved category 1 NCCN recommendations. A clinical trial comparing the two therapies in the setting of MTC has not yet been conducted. Only results from trials examining each agent versus placebo and preclinical studies are available to provide guidance for drug selection.

\section{Disclosure}

$\mathrm{R}$ Wein is a consultant for Bristol Myers Squibb. The authors have no other conflicts of interest to declare.

\section{References}

1. Kloos RT, Eng C, Evans DB, Francis GL, et al; American Thyroid Association Guidelines Task Force. Medullary thyroid cancer: management guidelines of the American Thyroid Association. Thyroid. 2009; 19:565-612.

2. Hundahl SA, Fleming ID, Fremgen AM, et al. A national cancer data base report on 53,856 cases of thyroid carcinoma treated in the US, 1985-1995. Cancer. 1998;83:2638-2648.

3. Dackiw AP. The surgical management of medullary thyroid cancer. Otolaryngol Clin North Am. 2010;43:365-374.

4. Vitale G, Caraglia M, Ciccarelli A, et al. Current approaches and perspectives in the therapy of medullary thyroid carcinoma. Cancer. 2001;91:1797-1808.

5. Pinchot SN, Kunnimalaiyaan M, Sippel RS, Chen H. Medullary thyroid carcinoma: targeted therapies and future directions. J Oncol. 2009;2009:183031.

6. Hart CD, De Boer RH. Profile of cabozantinib and its potential in the treatment of advanced medullary thyroid cancer. Onco Targets Ther. 2013;6:1-7. 
7. Nagilla M, Brown RL, Cohen EE. Cabozantinib for the treatment of advanced medullary thyroid cancer. Adv Ther. 2012;29:925-934.

8. Cerrato A, De Falco V, Santoro M. Molecular genetics of medullary thyroid carcinoma: the quest for novel therapeutic targets. $J \mathrm{Mol}$ Endocrinol. 2009;43:143-155.

9. Moura MM, Cavaco BM, Pinto AE, et al. Correlation of RET somatic mutations with clinicopathological features in sporadic medullary thyroid carcinomas. Br J Cancer. 2009;100:1777-1783.

10. Giunti S, Antonelli A, Amorosi A, Santarpia L. Cellular signaling pathway alterations and potential targeted therapies for medullary thyroid carcinoma. Int J Endocrinol. 2013;2013:803171.

11. Almeida MQ, Hoff AO. Recent advances in the molecular pathogenesis and targeted therapies of medullary thyroid carcinoma. Curr Opin Oncol. 2012;24:229-234.

12. Roman S, Mehta P, Sosa JA. Medullary thyroid cancer: early detection and novel treatments. Curr Opin Oncol. 2009;21:5-10.

13. National Comprehensive Cancer Network. Clinical Practice Guidelines in Oncology: Thyroid Carcinoma, Version 2. 2013

14. Witt RL. What is the treatment of the lateral neck in clinically localized sporadic medullary thyroid cancer? Laryngoscope. 2010;120 1286-1287.

15. Roman S, Lin R, Sosa JA. Prognosis of medullary thyroid carcinoma; demographic, clinical, and pathologic predictors of survival 1252 cases. Cancer. 2006;107:2134-2142.

16. Brierley J, Sherman E. The role of external beam radiation and targeted therapy in thyroid cancer. Semin Radiat Oncol. 2012;22:254-262.

17. Martinez SR, Beal SH, Chen A, Chen SL, Schneider PD. Adjuvant external beam radiation for medullary thyroid carcinoma. J Surg Oncol. 2010;102:175-178.

18. Skoura E. Depicting medullary thyroid cancer recurrence: the past and the future of nuclear medicine imaging. Int $J$ Endocrinol Metab. 2013;11:e8156, 1-8

19. Beuthien-Baumann B, Strumpf A, Zessin J, et al. Diagnostic impact of PET with 18F-FDG, 18F-DOPA and 3-O-methyl-6-[18F]fluoro-DOPA in recurrent or metastatic medullary thyroid carcinoma. Eur J Nucl Med Mol Imaging. 2007;34:1604-1609.

20. Hoegerle S, Altehoefer C, Ghanem N, et al. 18F-DOPA positron emission tomography for tumour detection in patient with medullary thyroid carcinoma and elevated calcitonin levels. Eur J Nucl Med. 2001;28 64-71.

21. Karras S, Pontikides N, Krassas GE. Pharmacokinetic evaluation of cabozantinib for the treatment of thyroid cancer. Expert Opin Drug Metab Toxicol. 2013;9:507-515.

22. Kurzrock R, Sherman SI, Ball DW, et al. Activity of XL184 (cabozantinib), an oral tyrosine inhibitor, in patients with medullary thyroid cancer. J Clin Oncol. 2011;29:2660-2666.

23. Sennino B, Ishiguro-Oonuma T, Wei Y, et al. Suppression of tumor invasion and metastasis by concurrent inhibition of c-Met and VEGF signaling in pancreatic neuroendocrine tumors. Cancer Discov. 2012;2 270-287.

24. Yakes FM, Chen J, Tan J, et al. Cabozantinib (XL184), a novel MET and VEGFR2 inhibitor, simultaneously suppresses metastasis, angiogenesis, and tumor growth. Mol Cancer Ther. 2011;10:2298-2308.

25. Bentzien F, Zuzow M, Heald N, et al. In vitro and in vivo activity of cabozantinib (XL184), an inhibitor of RET, MET, and VEGFR2, in model of medullary thyroid carcinoma. Thyroid. 2013;23:1569-1577.

26. Smith DC, Smith MR, Sweeney C, et al. Cabozantinib in patients with advanced prostate cancer: results of a Phase II randomized discontinuation trial. J Clin Oncol. 2013;31:412-419.

27. Memorial Sloan-Kettering Cancer Center. Cabozantinib in Patients With RET Fusion-Positive Advanced Non-Small Cell Lung Cancer. Available from: http://clinicaltrials.gov/show/NCT01639508. NLM identifier: NCT01639508. Accessed March 26, 2014.
28. Drilon A, Wang L, Hasanovic A, et al. Response to cabozantinib in patients with RET fusion-positive lung adenocarcinomas. Cancer Discov. 2013;3:630-635.

29. Salgia R, Hong DS, Camacho LH, et al. A Phase I dose-escalation study of the safety and pharmacokinetics (PK) of XL184, a VEGFR and MET kinase inhibitor, administered orally to patients (pts) with advanced malignancies. J Clin Oncol. 2007;25(Suppl 18):14031.

30. Elisei R, Schlumberger MJ, Müller SP, et al. Cabozantinib in progressive medullary thyroid cancer. J Clin Oncol. 2013;31:3639-3646.

31. Goldenberg MM. Pharmaceutical approval update. P T. 2013;38: $86-95$.

32. Robinson BG, Paz-Ares L, Krebs A, Vasselli J, Haddad R. Vandetanib $(100 \mathrm{mg})$ in patients with locally advanced or metastatic hereditary medullary thyroid cancer. J Clin Endocrinol Metab. 2010;95:2664-2671.

33. Wells SA Jr, Robinson BG, Gagel RF, et al. Vandetanib in patients with locally advanced or metastatic medullary thyroid cancer: a randomized, double-blind Phase III trial. J Clin Oncol. 2012;30:134-141.

34. Perez CA, Arango BA, Velez M, Raez LE, Santos ES. Emerging role of multikinase inhibitors for refractory thyroid cancer. Biologics. 2012;6: $257-265$.

35. Lam ET, Ringel MD, Kloos RT, et al. Phase II clinical trial of sorafenib in metastatic medullary thyroid cancer. J Clin Oncol. 2010;28: $2323-2330$

36. Schlumberger MJ, Elisei R, Bastholt L, et al. Phase II study of safety and efficacy of motesanib in patients with progressive or symptomatic, advanced or metastatic medullary thyroid cancer. J Clin Oncol. 2009;27: 3794-3801.

37. Goulart BH, Carr LL, Mankoff DA, et al. Phase II study of daily sunitinib in FDG-PET-positive, iodine-refractory differentiated thyroid cancer and metastatic medullary carcinoma of the thyroid with functional imaging correlation. Clin Cancer Res. 2010;16:5260-5268.

38. Cohen EE, Rosen LS, Vokes EE, et al. Axitinib is an active treatment for all histologic subtypes of advanced thyroid cancer: results from a Phase II study. J Clin Oncol. 2008;28:2323-2330.

39. de Groot JW, Zonnenberg BA, Quarles van Ufford-Mannesse P, et al. A Phase II trial of imatinib therapy for metastatic medullary thyroid carcinoma. J Clin Endocrinol Metab. 2007;92:3466-3469.

40. Frank-Raue K, Fabel M, Delorme S, Haberkorn U, Raue F. Efficacy of imatinib mesylate in advanced medullary thyroid carcinoma. Eur $J$ Endocrinol. 2007;157:215-220.

41. Verbeek HH, Alves MM, de Groot JW, et al. The effects of four different tyrosine kinase inhibitors on medullary and papillary thyroid cancer cells. J Clin Endocrinol Metab. 2011;96(6):E991-E995.

42. Brassard M, Rondeau G. Role of vandetanib in the management of medullary thyroid cancer. Biologics. 2012;6:59-66.

43. Viola D, Cappagli V, Elisei R. Cabozantinib (XL 184) for the treatment of locally advanced or metastatic progressive medullary thyroid cancer. Future Oncol. 2013;9(8):1083-1092.

44. Faggiano A, Ramundo V, Dicitore A, et al. Everolimus is an active agent in medullary thyroid cancer: a clinical and in vitro study. $J$ Cell Mol Med. 2012;16:1563-1572.

45. Starenki D, Singh NK, Jensen DR, Peterson FC, Park JI. Recombinant leukemia inhibitory factor suppresses human medullary thyroid carcinoma cell line xenografts in mice. Cancer Lett. 2013;339:144-151.

46. Samadi AK, Bazzill J, Zhang X, et al. Novel withanolides target medullary thyroid cancer through inhibition of both RET phosphorylation and the mammalian target of rapamycin pathway. Surgery. 2012;152:1238-1247.

47. Cakir M, Grossman AB. Medullary thyroid cancer: molecular biology and novel molecular therapies. Neuroendocrinology. 2009;90:323-348. 


\section{Publish your work in this journal}

Therapeutics and Clinical Risk Management is an international, peerreviewed journal of clinical therapeutics and risk management, focusing on concise rapid reporting of clinical studies in all therapeutic areas, outcomes, safety, and programs for the effective, safe, and sustained use of medicines. This journal is indexed on PubMed Central, CAS,

EMBase, Scopus and the Elsevier Bibliographic databases. The manuscript management system is completely online and includes a very quick and fair peer-review system, which is all easy to use. Visit http://www.dovepress.com/testimonials.php to read real quotes from published authors.

Submit your manuscript here: http://www.dovepress.com/therapeutics-and-clinical-risk-management-journal 